\title{
The costs of childhood ADHD extend into early adulthood
}

\author{
By Dr. Jessica Edwards
}

Earlier this year, Ebba Du Rietz and colleagues reported their findings from a large-scale, register-based study of the impact of childhood ADHD on healthcare use and costs in early adulthood. The researchers prospectively followed a large cohort of $>400,000$ young adults from 18 to 26 years-ofage. They obtained data on healthcare use from Swedish national patient and medication registers and then compared the annual costs per capita from multi-morbidity in those with and without a childhood diagnosis of ADHD. They found that a childhood diagnosis of ADHD was associated with greater long-term healthcare use for psychiatric and somatic disorders. This greater healthcare use conferred larger financial costs, with an average annual cost per capita of $€ 890$ for those with versus $€ 304$ for those without childhood ADHD. Interestingly, even those who no longer used healthcare services for ADHD in early adulthood (remitters) exhibited greater healthcare use and costs due to multi-morbidity than those without childhood ADHD. However, those who continued to have ADHD

Referring to:

Du Rietz, E., Jangmo, A., Kuja-Halkola, R., Chang, X., D'Onofrio, B.M., Ahnemark, E., Werner-Kiechle, T. \& Larsson, H. (2020), Trajectories of healthcare utilization and costs of psychiatric and somatic multimorbidity in adults with childhood ADHD: a prospective register-based study. J. Child Psychol. Psychiatr. doi: 10.111/ jcpp.13206. contact with healthcare services (persisters) had the greatest healthcare use and costs. Du Rietz et al. explain that prevention of multi-morbidity in people with childhood ADHD is needed both to benefit affected individuals and to alleviate the financial burden on society. 